\title{
Extraction of dihadron-jet correlations with rigorous flow-background subtraction in a multiphase transport model
}

\author{
Yuhui Zhu, ${ }^{1,2}$ Y. G. Ma* ${ }^{1}$ J. H. Chen,${ }^{1}$ G. L. Ma, ${ }^{1}$ S. Zhang, ${ }^{1}$ and C. Zhong ${ }^{1}$ \\ ${ }^{1}$ Shanghai Institute of Applied Physics, Chinese Academy of Sciences, Shanghai 201800, China \\ ${ }^{2}$ University of Chinese Academy of Sciences, Beijing 100049, China
}

(Dated: June 30, 2021)

\begin{abstract}
Dihadron azimuthal correlations in $\mathrm{Au}+\mathrm{Au}$ collisions at $\sqrt{S_{N N}}=200 \mathrm{GeV}$ have been explored by using a multi-phase transport (AMPT) model. In order to obtain the contributions from jetmedium interactions, the combined harmonic flow background is subtracted from the raw dihadron correlation functions. The signals are compared in three associated transverse momentum $\left(p_{T}^{a s s o c}\right)$ bins: $0.2-0.8 \mathrm{GeV} / \mathrm{c}, 0.8-1.4 \mathrm{GeV} / \mathrm{c}$ and $1.4-2.0 \mathrm{GeV} / \mathrm{c}$ from central to semi-peripheral geometries. The medium modifications are observed from changes in the signal shape and the relative jet contribution has been obtained within the change in the centrality from peripheral to central one. A strong $p_{T}^{a s s o c}$ dependence of the RMS width of jet correlation function is observed within the central geometry bin, i.e. $0-10 \%$.
\end{abstract}

PACS numbers: 25.75.Gz, 12.38.Mh, 24.85.+p

\section{INTRODUCTION}

Lattice quantum chromodynamics (QCD) calculations predicted a phase transition from hadron gas to a deconfined matter in ultra-relativistic heavy ion collisions [1, 2]. A hot and dense partonic matter, called Quark-Gluon Plasma (QGP), is formed in these collisions and is found to be strongly interacting.

In the literature, jet quenching [3] has been used as a signal of the QGP phase formation since it is interpreted as the evidence for the interaction between jets and the QGP medium. Dihadron azimuthal correlation acts as a probe for the study of jet quenching in high-energy heavy ion collisions. During the study of dihadron azimuthal correlation functions, an away-side double peak structure is observed in RHIC experiments at relatively high transverse momentum $\left(p_{T}\right)$ [4] 6 ], which is regarded as an evidence for jet quenching.

There are ongoing efforts within the RHIC comunity to study the inner mechanism of the jet-medium interaction through the dihadron correlation function. In this contest, many theoretical models have been put forward time to time such as shock wave model 7], gluon radiation model [8], medium-induced gluon bremsstrahlung [9, 10], waking the colored plasma and sonic Mach cones [11], sonic booms and diffusion wakes in thermal gauge-string duality [12], jet deflection [13] and strong parton cascade mechanism etc [14 18].

From the above studies, we know that dihadron azimuthal correlation signal is the key point to a solid physical interpretation. However, constructing a precise dihadron correlation background is a complex task. This is mainly because of the contributions from the oddorder harmonic flows, which are induced by initial ge-

\footnotetext{
*Author to whom all correspondence should be addressed: ygma@sinap.ac.cn
}

ometry fluctuations, to the away-side double peak structure [19, 20]. Theoretically, one can extract the harmonic flow coefficients and then reconstruct the high order harmonic background. However, due to the overestimation or underestimation of the non-flow contributions in our calculation method, it is hard to get an ideal flow coefficient. Therefore, more detailed analysis concerning the background construction is the need of the present time.

In this paper, we will employ two different methods for calculating background, which are discussed in detail in the upcoming section. These methods will be applied for the study of transverse momentum $\left(p_{T}\right)$ and centrality dependences $(0-10 \%, 20-40 \%$ and $50-80 \%)$ of dihadron azimuthal correlation functions in $200 \mathrm{GeV} / \mathrm{c} \mathrm{Au}+\mathrm{Au}$ collisions. Finally, the emphasis will be put on the study of jet-induced signals in different associated $p_{T}\left(p_{T}^{a s s o c}\right)$ bins from the central collisions.

The paper is organized as follows. Section II gives a brief introduction of the simulation model. Section III describes the two different analysis methods, which are used for background construction in the study of dihadron azimuthal correlation functions. The results and discussions are given in Section IV, which is followed by a summary in Section V.

\section{METHODOLOGY: A MULTI PHASE TRANSPORT MODEL (AMPT)}

A multi-phase transport model (AMPT) 21] is employed for the study of dihadron azimuthal correlations. The model has four main components for describing the physical processes in relativistic heavy-ion collisions: 1) the initial conditions from HIJING model [22], 2) partonic interactions modeled by Zhang's Parton Cascade model (ZPC) 23], 3) hadronization, and 4) hadronic rescattering simulated by A Relativistic Transport (ART) model 24]. The model works as follows: first, many excited strings initiated from HIJING are melted 
into partons in the string melting version of AMPT model 25] (abbr. "the Melting AMPT version"). Then, a simple quark coalescence model is used to combine the partons into hadrons. On the other hand, in the default version of AMPT model [26] (abbr. "the Default AMPT version"), minijet partons are recombined with their parent strings, when they stop interactions and the resulting strings are converted to hadrons via the Lund string fragmentation model [27]. It indicates that the Melting AMPT version undergoes a partonic phase much more than the Default AMPT version. The details of the AMPT model are available in a review paper [21] and previous works [21, 25, 28].

For the present study, the Melting AMPT version is used to perform the simulation for $200 \mathrm{GeV} / \mathrm{c} \mathrm{Au}+\mathrm{Au}$ collisions. Moreover, in order to concentrate on partonic stage interactions, final hadronic rescattering process is turned off as well.

\section{ANALYSIS METHOD}

The raw dihadron azimuthal correlations are obtained by following the experimental procedure [4, 5]. In the experiments, the azimuthal correlation is established between a high $p_{T}$ particle (trigger particle) and low $p_{T}$ particles (associated particles). In the present work, the particles in the range $p_{T}>2.5(\leq 2.5) \mathrm{GeV} / \mathrm{c}$ are specified as trigger particles (associated particles). Both the trigger and associated particles are required to be within a pseudo-rapidity window of $|\eta|<1.0$. The raw signal is obtained by accumulating pairs of trigger and associated particles into $\Delta \phi=\phi_{\text {assoc }}-\phi_{\text {trig }}$ distributions in the same event.

Normally, the dihadron combinational background can be described by the formula as follows [19, 20]:

$$
\langle f(\Delta \phi)\rangle_{e}=\left\langle\frac{N^{\text {trig }} \cdot N^{\text {assoc }}}{2 \pi}\right\rangle_{e}+\left\langle\frac{N^{\text {trig }} \cdot N^{\text {assoc }}}{2 \pi} \cdot 2 \sum_{n=1}^{+\infty} v_{n}^{\text {trig }} \cdot v_{n}^{a s s o c}\right\rangle_{e} \operatorname{cosn} \Delta \phi
$$

where suffix $e$ in $\langle\ldots\rangle_{e}$ stands for event-averaged quantity, while quantities without suffixes are for each event.

This is the first method, which is adopted in this study [20]. By applying the method, the initial geometry anisotropy $v_{n}^{r}$ is obtained with respect to the event plane extracted from the stage before the parton cascade. This will help to exclude as much non-flow contribution as possible. The event plane angle of each harmonic order is calculated by using the following formula:

$$
\Psi_{n}^{r}=\frac{1}{n}\left[\arctan \frac{\left\langle r^{n} \sin (n \phi)\right\rangle}{\left\langle r^{n} \cos (n \phi)\right\rangle}+\pi\right]
$$

where $r$ and $\phi$ are the polar coordinates of partons in the initial coordinate space. Then $v_{n}^{a s s o c}$ and $v_{n}^{\text {trig }}$ are calculated by using the formula:

$$
v_{n}^{r}=\left\langle\cos \left[n\left(\phi-\Psi_{n}^{r}\right)\right]\right\rangle
$$

where $\phi$ is the azimuthal angle in the final state momentum space. Here resolution correction for $\Psi_{n}^{r}$ is not necessary, since the event plane resolution from parton configuration space is considered to be nearly $100 \%$. As discussed earlier, this procedure effectively eliminates much nonflow contributions to harmonic flows 29]. From Ref. [20], we have seen that even in the most central collision $(b=0 \mathrm{fm})$, it is better to include higher order flow (up to fifth order) in background construction. Therefore, we have included up to fifth order flow contribution in background reconstruction.

However, here is a crucial point, which can not be neglected at the present stage. If one multiplies $v_{n}^{\text {assoc }}$ and $v_{n}^{\text {trig }}$ before being event averaged, two-particle $v_{n}$ correlation can lead to a contribution to the background, i.e. whether the factorization $\left(\left\langle v_{n}^{a s s o c} v_{n}^{\text {trig }}\right\rangle_{e}=\right.$ $\left.\left\langle v_{n}^{\text {assoc }}\right\rangle_{e}\left\langle v_{n}^{\text {trig }}\right\rangle_{e}\right)$ is held or not. In order to recover from the problem, the other modified form of the formula is also put forward in the literature [30, 31]. In the modified formula, systematic errors on $v_{n}$ are applied using background (Eq1) as the upper bound. It can be written as:

$$
\langle f(\Delta \phi)\rangle_{e}=\left\langle\frac{N^{\text {trig }} \cdot N^{\text {assoc }}}{2 \pi}\right\rangle_{e}+\left\langle\frac{N^{\text {trig }} \cdot N^{\text {assoc }}}{2 \pi}\right\rangle_{e} 2 \sum_{n=1}^{+\infty}\left\langle v_{n}^{\text {trig }}\right\rangle_{e}\left\langle v_{n}^{\text {assoc }}\right\rangle_{e} \cos n \Delta \phi .
$$

The difference between Eqns (11) and (4) contains two

kinds of contributions: 1. Flow fluctuation and its cor- 

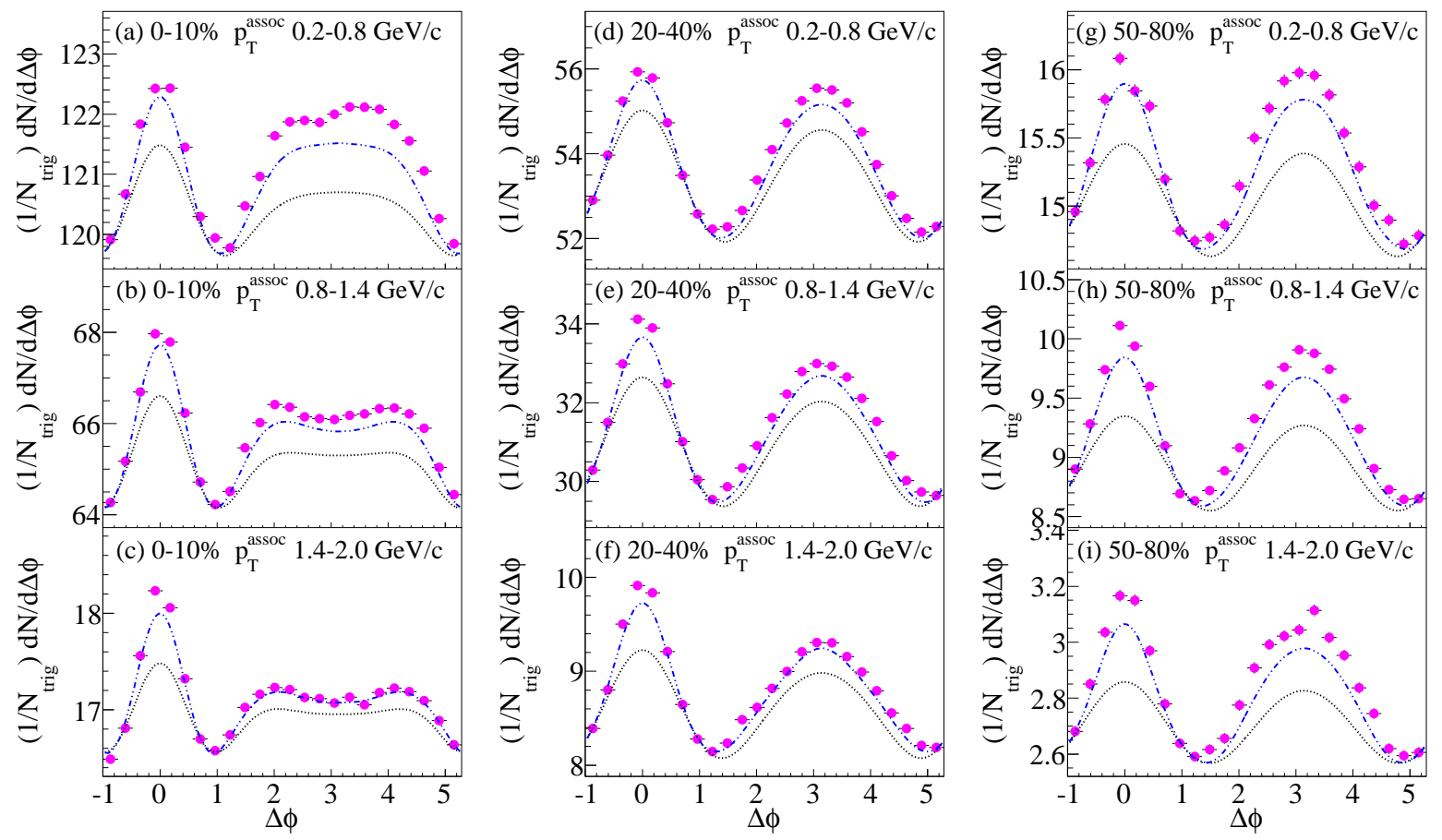

FIG. 1: (Color online) Dihadron correlation functions in $200 \mathrm{GeV} / \mathrm{c} \mathrm{Au}+\mathrm{Au}$ collisions derived in three $p_{T}^{a s s o c}$ bins as illustrated in the plots. (Left) 0-10\% centrality, (Middle) 20-40\% centrality, (Right) 50-80\% centrality . Solid circles are raw signals. Dash dotted blue lines are harmonic combinational backgrounds constructed using formula (1) and dash black lines are backgrounds constructed using formula (4).

relation from initial geometry asymmetry; 2. nonflow and its correlation. The first kind of contribution should be included in background, while the second kind should be excluded. However, neither background (Eq.1) and nor background (Eq.4) can meet the demand. The background (Eq.1) is found to overestimate background by including nonflow contribution, while background (Eq.4) underestimates the background by throwing away the background from flow fluctuation and its correlation. It is worth mentioning that there have been some efforts to solve the crucial problem on decomposing flow, flow fluctuation, and nonflow 32.

Although an ideal background is hard to be obtained, however, one can use the two formulas from Eqns. (1) and (4) as the upper and lower limits of the background to get a reasonable range of jet-medium contribution. Following the same method, background is reconstructed using Eqns. (1) and (4) as the upper and lower limits for dihadron background. Correspondingly, they are marked as "background (Eq.1)" and "background (Eq.4)" in the figures. The detailed values of parameters $\left\langle v_{1}^{\text {trig }}\right\rangle$, $\left\langle v_{1}^{a s s o c}\right\rangle$ and $\left\langle v_{1}^{\text {trig }} v_{1}^{a s s o c}\right\rangle$ used in these two methods of background extractions are listed in appendix A.

From the Eqns. (1) and (4), we have observed that the normalization factor of the background should be $\left\langle\frac{N^{t r i g} \cdot N^{a s s o c}}{2 \pi}\right\rangle_{e}$ theoretically. Another method is to use
ZYAM scheme (A Zero Yield At Minimum) for adjusting this factor to best match the signal with experimental findings. However, we have checked the results from both way and found that the difference between the theoretical normalization factor and the ZYAM adjustment is less than $3 \%$.

\section{RESULTS AND DISCUSSIONS}

\section{A. Two Particle Azimuthal Correlation in Three Centrality Bins}

Fig. 1 shows the dihadron azimuthal correlations (both raw and combined harmonic background) with different centrality bins from left to right panels. Each panel from top to bottom is displaying the results with different $p_{T}^{a s s o c}$ bins. For the better understanding, two backgrounds have been drawn simultaneously.

Firstly, an obvious difference in shape of background in different centralities is observed. The central 0-10\% events have flatter or even double-peak shape background, while $20-40 \%$ and $50-80 \%$ ones have a singleGaussian shape background. This is due to the different propagation properties of harmonic flows for different centralities. The existence of the hot dense matter (QGP) propagates the initial geometry irregularities to a larger extent. In Ref. [29], $p_{T}$ dependencies of $v_{2}$ and $v_{3}$ 

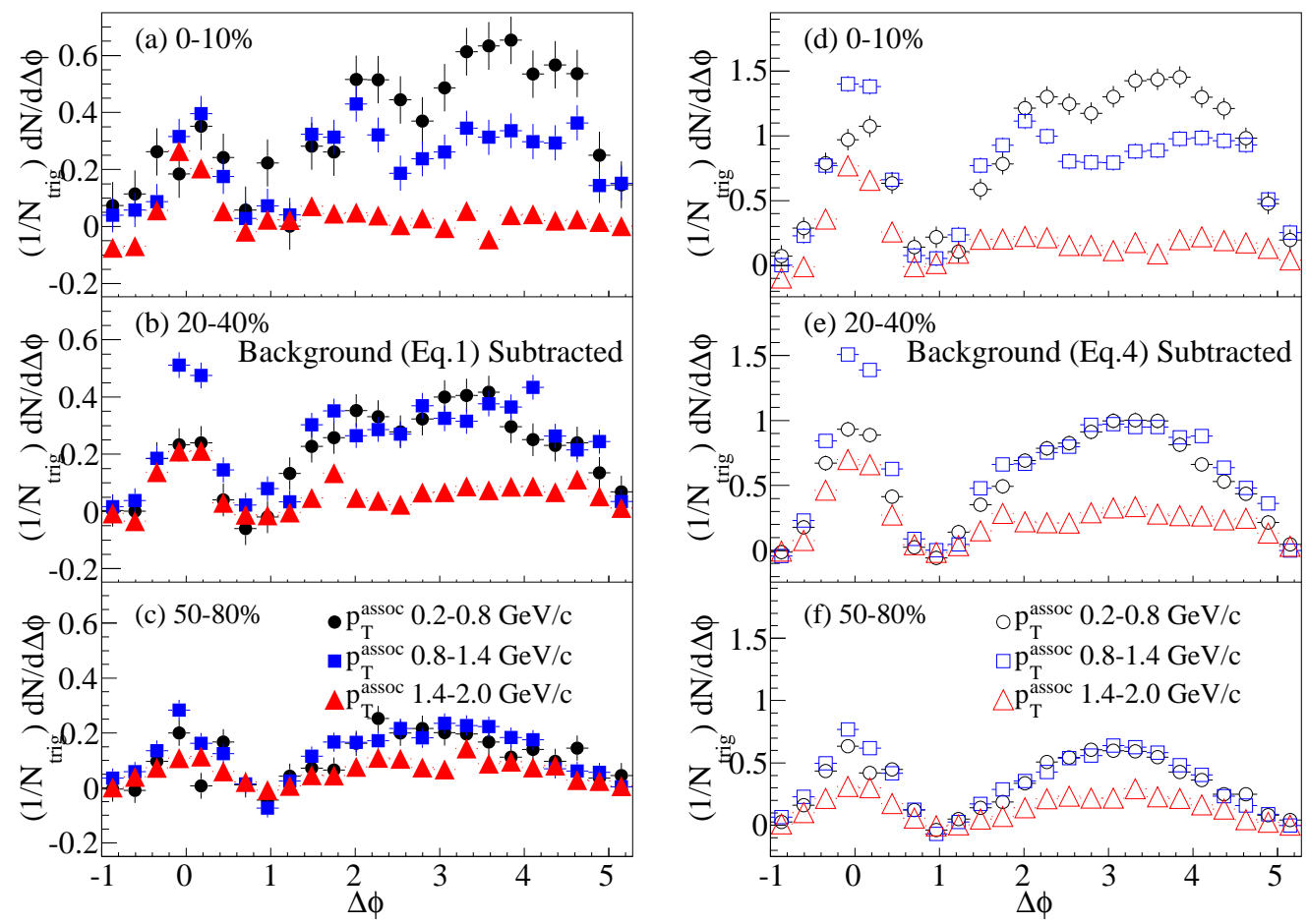

FIG. 2: (Color online) Background subtracted signals in three $p_{T}^{a s s o c}$ bins for three centralities. Panels (a), (b), (c) stand for the signals with background (Eq.1) subtracted; panels (d), (e), (f) stands for the signals after background (Eq.4) subtracted. See texts for details.
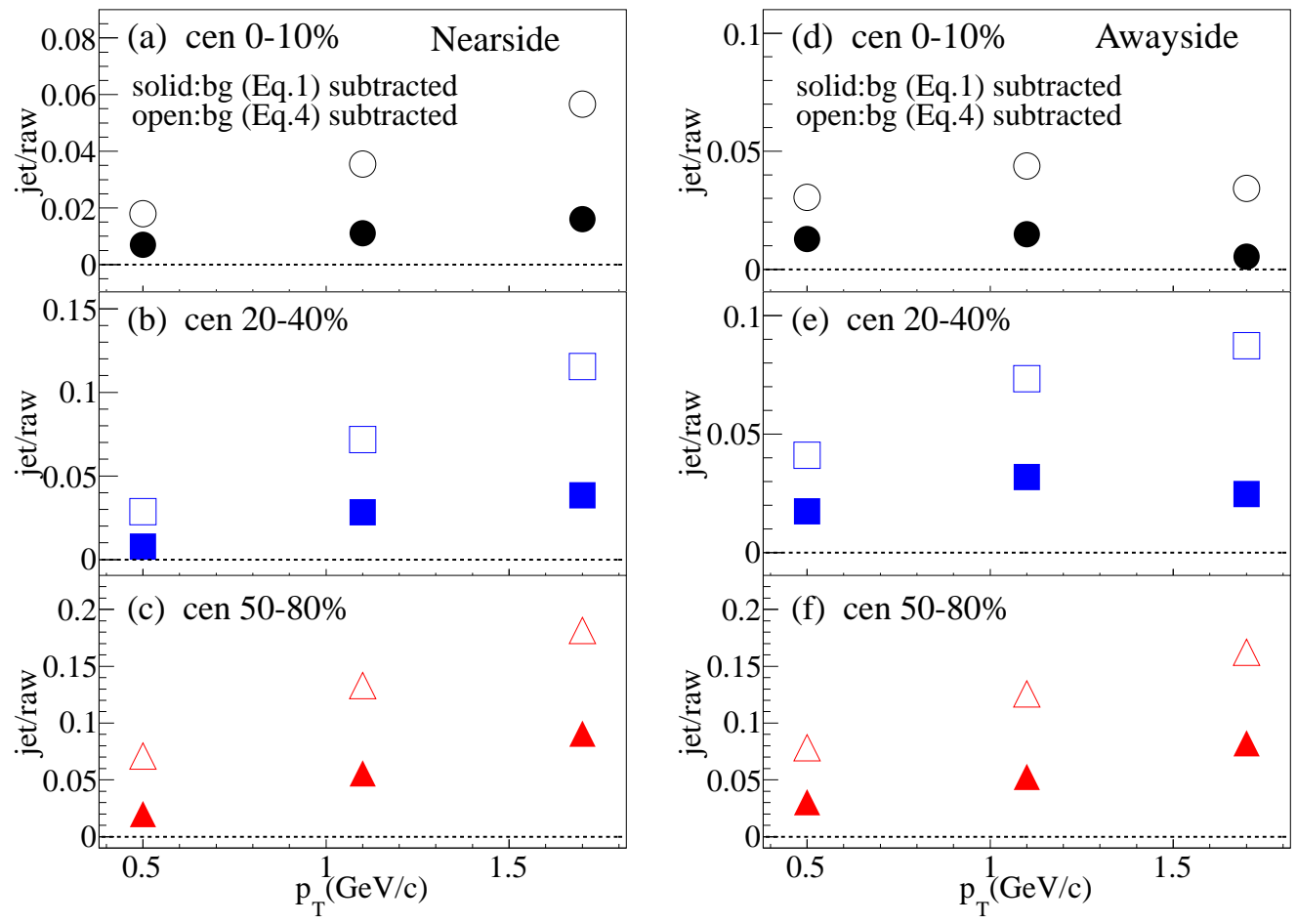

FIG. 3: (Color online) Jet relative contributions as a function of $p_{T}^{a s s o c}$ in three centrality bins for near-side (panels:(a),(b),(c)) and away-side (panels:(d),(e),(f)). 
show a stronger dependence especially in large $p_{T}$ range, which makes $v_{3}$ to increase more rapidly. Furthermore, $v_{3}$ contribution is strongly affected with the change in the geometry from central to peripheral one. This leads to the change of combinational background shape to flatter or double peak in the $p_{T}$ range from 1.4 to $2.0 \mathrm{GeV} / \mathrm{c}$. For the two backgrounds, they tend to have similar shapes and just differ a little bit in magnitudes.

Secondly, we have found a seemingly changing trend of the signal using either background. Therefore, we need to subtract the background and further study the jet-medium contribution. The corresponding plots are shown in Fig. 2. The panels (a) to (c) ((d) to (f)) represent the results with background Eq.1 (Eq.4) subtracted. From the global comparison of these two panel sets, an obvious change in signal shape has been observed. The signals in more central events $(0-10 \%)$ or higher $p_{T}^{\text {assoc }}$ range (eg. 1.4-2.0 GeV/c) tend to have flatter shape signal, while peripheral $50-80 \%$ events almost show a single-peak shape signal. This difference is consistent with the medium modification picture. The existence of QGP strongly modifies jets, which makes the correlation shape flatter (or more broaden) in the most central collisions. In addition, it also suppresses higher $p_{T}$ particles. On the other hand, if jets interactions with surrounding medium particles are less (peripheral collisions), the correlation shape will tend to be single Gaussian. Moreover, the previous results indicate that there is also some contributions from hot spots, when the jet production is switched off [20].

For the further analysis, a quantity named "jet relative contribution" is used to represent the contribution of jet-medium correlation in total dihadron correlation function. It is defined as the ratio of jet-medium correlation function yield to the raw dihadron correlation yield (including flow background). These jet relative contributions in different centrality and $p_{T}^{a s s o c}$ bins are calculated and results are shown in Fig. 3. The panels (a), (b), (c) in Fig. 3 are for near-side and panels (d), (e), (f) are for away-side jet relative contribution. The jet relative contributions using two different backgrounds are drawn together for providing an upper and lower limit. From panel (d), one can see that the away-side jet relative contribution in central $0-10 \%$ events drops in $p_{T}^{a s s o c}$ range from 1.4 to $2.0 \mathrm{GeV} / \mathrm{c}$, which is different from the case of near-side contribution (panel (a)). This is consistent with the high $p_{T}$ suppression picture in QGP. The quantitative values are provided in Table I and II. In conclusion, the away-side jet relative contribution is less than $5 \%$ in central $0-10 \%$ events.

\section{B. $p_{T}^{a s s o c}$ dependence of jet-medium contribution in central $0-10 \%$ collisions}

As we have already observed the modification to the correlation function by jet-medium interactions in Fig. 2 and 3, now we would like to focus on central 0-10\% events
TABLE I: Near-side Jet-medium Contribution

\begin{tabular}{llll}
\hline \hline & $0.2-0.8 \mathrm{GeV} / \mathrm{c}$ & $0.8-1.4 \mathrm{GeV} / \mathrm{c}$ & $1.4-2.0 \mathrm{GeV} / \mathrm{c}$ \\
$0-10 \%$ & $0.7 \%-1.8 \%$ & $1.1 \%-3.5 \%$ & $1.6 \%-5.7 \%$ \\
$20-40 \%$ & $0.8 \%-2.9 \%$ & $2.8 \%-7.2 \%$ & $3.8 \%-11.5 \%$ \\
$50-80 \%$ & $2.0 \%-7.1 \%$ & $5.2 \%-13.3 \%$ & $9.1 \%-18.1 \%$ \\
\hline \hline
\end{tabular}

TABLE II: Away-side Jet-medium Contribution

\begin{tabular}{llll}
\hline \hline & $0.2-0.8 \mathrm{GeV} / \mathrm{c}$ & $0.8-1.4 \mathrm{GeV} / \mathrm{c}$ & $1.4-2.0 \mathrm{GeV} / \mathrm{c}$ \\
$0-10 \%$ & $1.3 \%-3.1 \%$ & $1.5 \%-4.4 \%$ & $0.5 \%-3.4 \%$ \\
$20-40 \%$ & $1.7 \%-4.1 \%$ & $3.2 \%-7.3 \%$ & $2.5 \%-8.7 \%$ \\
$50-80 \%$ & $3.0 \%-7.8 \%$ & $5.2 \%-12.6 \%$ & $8.2 \%-16.2 \%$ \\
\hline \hline
\end{tabular}

for more detailed analysis. This is important because the modifications are the most obvious near the central geometry. For this purpose, the whole $p_{T}^{a s s o c}$ range (from $0.2 \mathrm{GeV} / \mathrm{c}$ to $2.4 \mathrm{GeV} / \mathrm{c}$ ) is divided into much fine bins (with a bin width of $0.2 \mathrm{GeV} / \mathrm{c}$ ). The results are shown in Fig. 4, where total $11 p_{T}^{a s s o c}$ bins are taken into consideration for central events. As is seen clearlt from Fig. 4 that the background (Eq.1) almost overlaps with raw signal in high $p_{T}$ range (larger than $1.6 \mathrm{GeV} / \mathrm{c}$ ), so we only pick out $7 p_{T}$ bin results for further analysis. For background (Eq.2) case, all the real signals are extracted.

The extracted values of background (Eq. 1) and background (Eq. 4) from Fig. 4 are displayed in the left and middle panels of Fig. 5. The different colored and type of the symbols represent the values extracted from different $p_{T}^{a s s o c}$ bins. For background (Eq.1) case (panels (a), (b)), we have found that the signal shape becomes flatter and flatter towards the high $p_{T}^{a s s o c}$. For background (Eq.4) case (panels (c) to (e)), a clear evolution from a flat or seemingly single peak structure to a double peak structure with $p_{T}^{a s s o c}$ is obtained. This is because of the unsubtracted $v_{3}$ fluctuation and correlation. The RMS and jet relative contribution, which are extracted from the left and middle panels, are shown in the right panels (f) and (g) of Fig. 5. The $p_{T}^{\text {assoc }}$ dependence of RMS also shows the similar evolutions of away-side signal shape for both the cases. The reasonable range of jet relative contribution is shown in the panel $(\mathrm{g})$. These results suggest the importance of jet-medium interactions, and may help elucidate the mechanisms of jet energy loss in the QGP.

\section{SUMMARY}

In summary, we have studied the dihadron azimuthal correlation functions using a multi-phase transport (AMPT) model in $200 \mathrm{GeV} / \mathrm{c} \mathrm{Au}+\mathrm{Au}$ collisions for different centralities of $0-10 \%, 20-40 \%$ and $50-80 \%$ and different $p_{T}^{a s s o c}$ bins. We have obtained the harmonic flows with less nonflow effect and constructed the combined harmonic flow background using two formulas as 

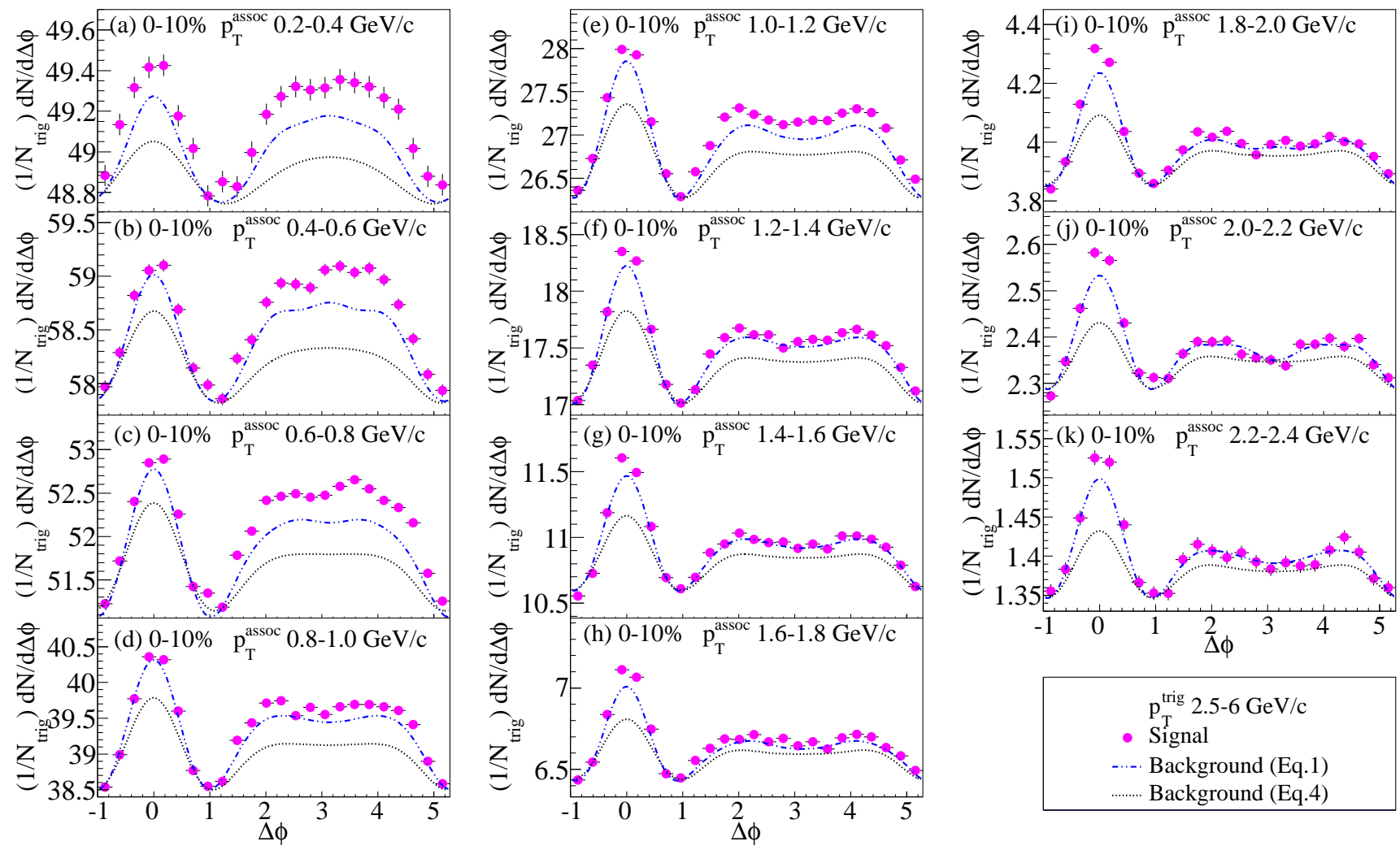

FIG. 4: (Color online) Dihadron correlation functions for different $p_{T}^{a s s o c}$ bins in $200 \mathrm{GeV} / \mathrm{c} \mathrm{Au}+\mathrm{Au}$ collisions at $0-10 \%$ centrality. From upper-left to bottom-right, the $p_{T}^{a s s o c}$ are separated into 11 bins with $0.2 \mathrm{GeV} / \mathrm{c}$ bin width. Solid circles are raw signals, dash-dotted blue curves are the combinational background (Eq.1) cases and dotted black curves are the combinational background (Eq.4) cases.
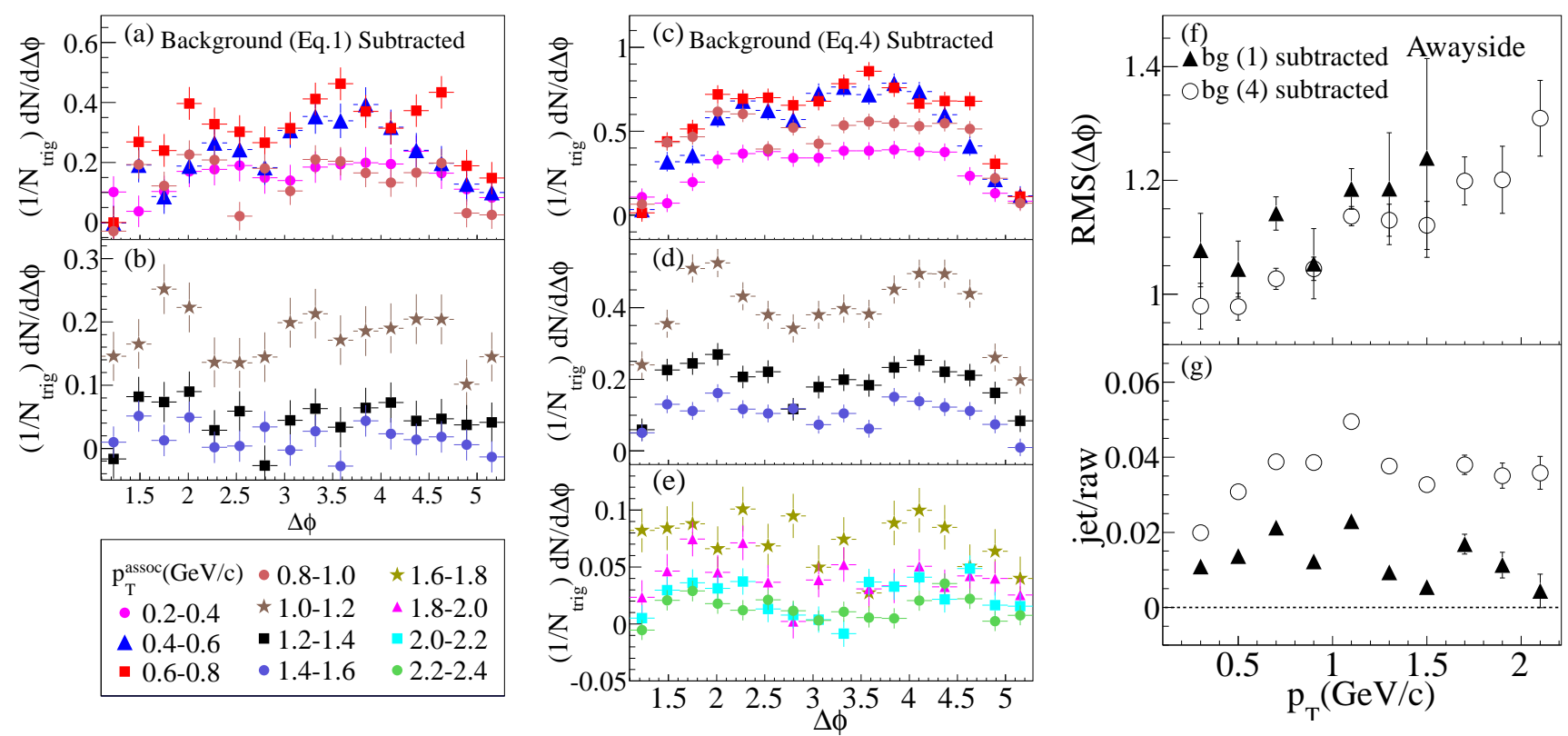

FIG. 5: (Color online) Panels (a), (b): background subtracted away-side signals for seven different $p_{T}^{a s s o c}$ bins for background (Eq.1) case; Panels (c), (d), (e): away-side signals for eleven different bins for background (Eq.4) case; Panels (f), (g): $p_{T}^{a s s o c}$ dependences of RMS width and jet relative contribution. 
reasonable upper and lower limits. Although the backgrounds calculated by two formulas differ in magnitude, the physics information is quite similar for both cases.

The evolution of real signal shape and away-side jet relative contribution with the increase of $p_{T}^{a s s o c}$ and centrality is consistent with the fact that the high $p_{T}$ particles are strongly modified by the hot dense medium and the hot dense medium formation is weaker in more peripheral collisions.

The jet contribution percentage in the raw dihadron correlation function is relatively small. For the most central events $(0-10 \%)$, it is less than $5 \%$. For a reliable extraction of the jet-correlation yields, a precise understanding of the harmonic flow background to within a few percent is required. However, the jet-correlation shape is robust against a large variation in the background sub- traction. We have carried out a comprehensive study of jet-medium contribution as a function of $p_{T}^{a s s o c}$ in fine bins. We observe an evolution of the correlation signal with increasing $p_{T}^{a s s o c}$, from a single Gaussian to a flat or even double-peaked shape. The away-side correlation function RMS increases with $p_{T}^{a s s o c}$, even for our conservative choices of the two significantly different flow backgrounds. These results suggest the importance of jetmedium interactions, and may help elucidate the mechanisms of jet energy loss in the QGP.

This work was supported in part by the National Natural Science Foundation of China under contract Nos. 11035009, 11220101005, 10979074, 11105207, 11175232, U1232206 and the Knowledge Innovation Project of the Chinese Academy of Sciences under Grant No. KJCX2EW-N01.
[1] F. R. Brown, F. P. Butler, Hong Chen, N. H. Christ, Zhihua Dong, W. Schaffer, L. I. Unger, and A. Vaccarino, Phys. Rev. Lett. 65, 2491 (1990).

[2] I. Arsene et al., Nucl. Phys. A757, 1 (2005); B. B. Back et al. (PHOBOS Collaboration), ibid. A757, 28 (2005); J. Adams et al. (STAR Collaboration), ibid. A757, 102 (2005); S. S. Adcox et al. (PHENIX Collaboration), ibid. A757, 184 (2005).

[3] X.-N. Wang and M. Gyulassy, Phys. Rev. Lett. 68, 1480 (1992).

[4] J. Adams et al. (STAR Collaboration), Phys. Rev. Lett. 95, 152301 (2005).

[5] S.S. Adler et al. (PHENIX Collaboration), Phys. Rev. Lett. 97, 052301 (2006).

[6] B. I. Abelev et al., Phys. Rev. Lett. 102, 052302 (2009).

[7] H. Stoecker, Nucl. Phys. A 750, 121 (2005); J. Casalderrey-Solana et al., J. Phys. Conf. Ser. 27, 22 (2005); Nucl. Phys. A 774, 577 (2006).

[8] V. Koch, A. Majumder, Xin-Nian Wang, Phys. Rev. Lett. 96, 172302 (2006).

[9] I. Vitev, Phys. Lett. B 630, 78 (2005).

[10] A. D. Polosa and C. A. Salgado, Phys. Rev. C 75, 041901 (R) (2007).

[11] J. Ruppert, B. Müller, Phys. Lett. B 618, 123 (2005); R. B. Neufeld, B. Muller and J. Ruppert, Phys. Rev. C 78, 041901 (2008).

[12] S. S. Gubser, S. S. Pufu, Phys. Rew. Lett. 100, 012301 (2008).

[13] N. Armesto, C. A. Salgado, and U. A. Wiedemann, Phys. Rew. C 72, 064910 (2005).

[14] Y. G. Ma, J. Phys. G 32, S373 (2006).

[15] G. L. Ma, S. Zhang, Y. G. Ma et al., Phys. Lett. B 641, 362 (2006).

[16] G. L. Ma, Y. G. Ma, S. Zhang et al., Phys. Lett. B 647, 122 (2007).

[17] G. L. Ma, S. Zhang, Y. G. Ma et al.,
arXiv:nucl-th/0610088

[18] S. Zhang, G. L. Ma, Y. G. Ma et al., Phys. Rev. C 76, 014904 (2007).

[19] J. Xu and C. M. Ko, Phys. Rev. C 83, 021903 (2011).

[20] G. L. Ma and X. N. Wang, Phys. Rev. Lett. 106, 162301 (2011).

[21] Z. W. Lin, C. M. Ko, B. A. Li, B. Zhang, S. Pal, Phys. Rev. C 72, 064901 (2005).

[22] X.-N. Wang and M. Gyulassy, Phys. Rev. D 44, 3501 (1991); M. Gyulassy and X.-N. Wang, Comput. Phys. Commun. 83, 307 (1994).

[23] B. Zhang, Comput. Phys. Commun. 109, 193 (1998).

[24] B. A. Li and C. M. Ko, Phys. Rev. C 52, 2037 (1995).

[25] Z. W. Lin, C. M. Ko, Phys. Rev. C 65, 034904 (2002); Z. W. Lin, C. M. Ko et al., Phys. Rev. Lett. 89, 152301 (2002).

[26] B. Zhang, C. M. Ko et al., Phys. Rev. C 61, 067901 (2000).

[27] B. Andersson, G. Gustafson, G. Ingelman, T. Sjöstrand, Phys. Rep. 97, 31 (1983).

[28] J. H. Chen, Y. G. Ma, G. L. Ma et al., Phys. Rev. C 74, 064902 (2006).

[29] L. Han, G. L. Ma, Y. G. Ma et al., Phys. Rev. C 84, 064907 (2011).

[30] M. M. Aggarwal et al. (STAR Collaboration), Phys. Rev. C 82, 024912 (2010).

[31] G. Agakishiev et al. (STAR Collaboration), Phys. Rev. C 85, 014903 (2012).

[32] L. Yi, F. Q. Wang, and A. H. Tang, arXiv:1101.4646 [nucl-ex]; L. Xu, L. Yi, D. Kikola, J. Konzer, F. Q. Wang, and W. Xie, arXiv:1204.2815 [nucl-ex].

Appendix A: List of values of flow parameters 
TABLE III: $\left\langle v_{1}^{\text {trig }}\right\rangle\left\langle v_{1}^{\text {assoc }}\right\rangle\left\langle v_{1}^{\text {trig }} v_{1}^{\text {assoc }}\right\rangle$

\begin{tabular}{llllllllll}
\hline \hline & \multicolumn{3}{c}{$0-10 \%$} \\
$p_{T}^{\text {assoc }} \mathrm{range}$ & $\left\langle v_{1}^{\text {trig }}\right\rangle$ & $\left\langle v_{1}^{\text {assoc }}\right\rangle$ & $\left\langle v_{1}^{\text {trig }} v_{1}^{\text {assoc }}\right\rangle$ & $\left\langle v_{1}^{\text {trig }}\right\rangle$ & \multicolumn{2}{c}{$\begin{array}{l}20-40 \% \\
\left\langle v_{1}^{\text {assoc }}\right\rangle\end{array}$} & $\left\langle v_{1}^{\text {trig }} v_{1}^{\text {assoc }}\right\rangle$ & $\left\langle v_{1}^{\text {trig }}\right\rangle$ & \multicolumn{2}{c}{$50-80 \%$} & $\left\langle v_{1}^{\text {assoc }}\right\rangle$ & $\left\langle v_{1}^{\text {trig }} v_{1}^{\text {assoc }}\right\rangle$ \\
$0.2-0.4 \mathrm{GeV} / \mathrm{c}$ & -0.025871 & 0.003891 & -0.000697 & -0.002960 & 0.001342 & -0.000942 & -0.005993 & 0.000993 & -0.001940 \\
$0.4-0.6 \mathrm{GeV} / \mathrm{c}$ & -0.025871 & 0.004727 & -0.001506 & -0.002960 & 0.001394 & -0.001624 & -0.005993 & 0.000642 & -0.002396 \\
$0.6-0.8 \mathrm{GeV} / \mathrm{c}$ & -0.025871 & 0.003769 & -0.001587 & -0.002960 & 0.001312 & -0.001456 & -0.005993 & -0.000149 & -0.002416 \\
$0.8-1.0 \mathrm{GeV} / \mathrm{c}$ & -0.025871 & 0.001927 & -0.001085 & -0.002960 & 0.000702 & -0.001577 & -0.005993 & -0.000068 & -0.002329 \\
$1.0-1.2 \mathrm{GeV} / \mathrm{c}$ & -0.025871 & -0.000342 & -0.000595 & -0.002960 & 0.000570 & -0.001468 & -0.005993 & 0.000488 & -0.003082 \\
$1.2-1.4 \mathrm{GeV} / \mathrm{c}$ & -0.025871 & -0.001999 & -0.000035 & -0.002960 & -0.000051 & -0.000855 & -0.005993 & -0.001384 & -0.003353 \\
$1.4-1.6 \mathrm{GeV} / \mathrm{c}$ & -0.025871 & -0.004586 & 0.000807 & -0.002960 & -0.000390 & -0.000036 & -0.005993 & -0.003510 & -0.003151 \\
$1.6-1.8 \mathrm{GeV} / \mathrm{c}$ & -0.025871 & -0.007725 & 0.001316 & -0.002960 & -0.001960 & 0.000864 & -0.005993 & 0.002034 & -0.003610 \\
$1.8-2.0 \mathrm{GeV} / \mathrm{c}$ & -0.025871 & -0.010687 & 0.002172 & -0.002960 & -0.003233 & 0.001257 & -0.005993 & 0.000679 & -0.003284 \\
$2.0-2.2 \mathrm{GeV} / \mathrm{c}$ & -0.025871 & -0.012145 & 0.003433 & -0.002960 & -0.002566 & 0.001859 & -0.005993 & -0.002832 & -0.003654 \\
$2.2-2.4 \mathrm{GeV} / \mathrm{c}$ & -0.025871 & -0.016067 & 0.003415 & -0.002960 & -0.003811 & 0.002671 & -0.005993 & -0.005312 & -0.004666 \\
\hline \hline
\end{tabular}

TABLE IV: $\left\langle v_{2}^{\text {trig }}\right\rangle\left\langle v_{2}^{\text {assoc }}\right\rangle\left\langle v_{2}^{\text {trig }} v_{2}^{\text {assoc }}\right\rangle$

\begin{tabular}{llllllllll}
\hline \hline & \multicolumn{3}{c}{$0-10 \%$} & & & & & & $50-40 \%$ \\
$p_{T}^{\text {assoc }}$ range & $\left\langle v_{2}^{\text {trig }}\right\rangle$ & $\left\langle v_{2}^{\text {assoc }}\right\rangle$ & $\left\langle v_{2}^{\text {trig }} v_{2}^{\text {assoc }}\right\rangle$ & $\left\langle v_{2}^{\text {trig }}\right\rangle$ & $\left\langle v_{2}^{\text {assoc }}\right\rangle$ & $\left\langle v_{2}^{\text {trig }} v_{2}^{\text {assoc }}\right\rangle$ & $\left\langle v_{2}^{\text {trig }}\right\rangle$ & $\left\langle v_{2}^{\text {assoc }}\right\rangle$ & $\left\langle v_{2}^{\text {trig }} v_{2}^{\text {assoc }}\right\rangle$ \\
$0.2-0.4 \mathrm{GeV} / \mathrm{c}$ & 0.073775 & 0.016994 & 0.001957 & 0.188455 & 0.041964 & 0.009125 & 0.164376 & 0.052629 & 0.012003 \\
$0.4-0.6 \mathrm{GeV} / \mathrm{c}$ & 0.073775 & 0.031763 & 0.003030 & 0.188455 & 0.070599 & 0.015442 & 0.164376 & 0.081647 & 0.018957 \\
$0.6-0.8 \mathrm{GeV} / \mathrm{c}$ & 0.073775 & 0.044547 & 0.004151 & 0.188455 & 0.095192 & 0.021016 & 0.164376 & 0.103296 & 0.024413 \\
$0.8-1.0 \mathrm{GeV} / \mathrm{c}$ & 0.073775 & 0.053954 & 0.004852 & 0.188455 & 0.114463 & 0.025449 & 0.164376 & 0.120846 & 0.028254 \\
$1.0-1.2 \mathrm{GeV} / \mathrm{c}$ & 0.073775 & 0.060495 & 0.005207 & 0.188455 & 0.129821 & 0.029124 & 0.164376 & 0.133234 & 0.032168 \\
$1.2-1.4 \mathrm{GeV} / \mathrm{c}$ & 0.073775 & 0.064775 & 0.006021 & 0.188455 & 0.141029 & 0.031751 & 0.164376 & 0.143701 & 0.035081 \\
$1.4-1.6 \mathrm{GeV} / \mathrm{c}$ & 0.073775 & 0.067712 & 0.006900 & 0.188455 & 0.149935 & 0.034043 & 0.164376 & 0.151426 & 0.038436 \\
$1.6-1.8 \mathrm{GeV} / \mathrm{c}$ & 0.073775 & 0.068329 & 0.007220 & 0.188455 & 0.156176 & 0.035729 & 0.164376 & 0.152973 & 0.038768 \\
$1.8-2.0 \mathrm{GeV} / \mathrm{c}$ & 0.073775 & 0.068417 & 0.007574 & 0.188455 & 0.160479 & 0.037796 & 0.164376 & 0.155515 & 0.040527 \\
$2.0-2.2 \mathrm{GeV} / \mathrm{c}$ & 0.073775 & 0.066030 & 0.007593 & 0.188455 & 0.163173 & 0.038348 & 0.164376 & 0.159956 & 0.043669 \\
$2.2-2.4 \mathrm{GeV} / \mathrm{c}$ & 0.073775 & 0.064178 & 0.008366 & 0.188455 & 0.163583 & 0.038770 & 0.164376 & 0.160305 & 0.043453 \\
\hline \hline
\end{tabular}

TABLE V: $\left\langle v_{3}^{\text {trig }}\right\rangle\left\langle v_{3}^{\text {assoc }}\right\rangle\left\langle v_{3}^{\text {trig }} v_{3}^{\text {assoc }}\right\rangle$

\begin{tabular}{lllllllllll}
\hline \hline & \multicolumn{3}{c}{$0-10 \%$} & & & & & $50-40 \%$ \\
$p_{T}^{\text {assoc }}$ range & $\left\langle v_{3}^{\text {trig }}\right\rangle$ & $\left\langle v_{3}^{\text {assoc }}\right\rangle$ & $\left\langle v_{3}^{\text {trig }} v_{3}^{\text {assoc }}\right\rangle$ & $\left\langle v_{3}^{\text {trig }}\right\rangle$ & $\left\langle v_{3}^{\text {assoc }}\right\rangle$ & $\left\langle v_{3}^{\text {trig }} v_{3}^{\text {assoc }}\right\rangle$ & $\left\langle v_{3}^{\text {trig }}\right\rangle$ & $\left\langle v_{3}^{\text {assoc }}\right\rangle$ & $\left\langle v_{3}^{\text {trig }} v_{3}^{\text {assoc }}\right\rangle$ \\
$0.2-0.4 \mathrm{GeV} / \mathrm{c}$ & 0.100672 & 0.004909 & 0.001118 & 0.098937 & 0.010104 & 0.001703 & 0.057041 & 0.012910 & 0.002508 \\
$0.4-0.6 \mathrm{GeV} / \mathrm{c}$ & 0.100672 & 0.015469 & 0.002593 & 0.098937 & 0.021127 & 0.003401 & 0.057041 & 0.019854 & 0.003226 \\
$0.6-0.8 \mathrm{GeV} / \mathrm{c}$ & 0.100672 & 0.028102 & 0.004178 & 0.098937 & 0.032916 & 0.005453 & 0.057041 & 0.027854 & 0.004895 \\
$0.8-1.0 \mathrm{GeV} / \mathrm{c}$ & 0.100672 & 0.040093 & 0.006086 & 0.098937 & 0.043845 & 0.007427 & 0.057041 & 0.033936 & 0.006076 \\
$1.0-1.2 \mathrm{GeV} / \mathrm{c}$ & 0.100672 & 0.050931 & 0.007859 & 0.098937 & 0.053737 & 0.008962 & 0.057041 & 0.039496 & 0.006865 \\
$1.2-1.4 \mathrm{GeV} / \mathrm{c}$ & 0.100672 & 0.059100 & 0.008760 & 0.098937 & 0.061137 & 0.010424 & 0.057041 & 0.045423 & 0.008008 \\
$1.4-1.6 \mathrm{GeV} / \mathrm{c}$ & 0.100672 & 0.066054 & 0.009964 & 0.098937 & 0.068773 & 0.011919 & 0.057041 & 0.048002 & 0.008809 \\
$1.6-1.8 \mathrm{GeV} / \mathrm{c}$ & 0.100672 & 0.071490 & 0.010496 & 0.098937 & 0.074461 & 0.013307 & 0.057041 & 0.051733 & 0.010559 \\
$1.8-2.0 \mathrm{GeV} / \mathrm{c}$ & 0.100672 & 0.075416 & 0.011367 & 0.098937 & 0.077711 & 0.013558 & 0.057041 & 0.054062 & 0.010938 \\
$2.0-2.2 \mathrm{GeV} / \mathrm{c}$ & 0.100672 & 0.076608 & 0.012745 & 0.098937 & 0.082178 & 0.014395 & 0.057041 & 0.058495 & 0.008238 \\
$2.2-2.4 \mathrm{GeV} / \mathrm{c}$ & 0.100672 & 0.078378 & 0.013210 & 0.098937 & 0.085642 & 0.014619 & 0.057041 & 0.061674 & 0.014042 \\
\hline \hline
\end{tabular}


TABLE VI: $\left\langle v_{4}^{\text {trig }}\right\rangle\left\langle v_{4}^{\text {assoc }}\right\rangle\left\langle v_{4}^{\text {trig }} v_{4}^{\text {assoc }}\right\rangle$

\begin{tabular}{|c|c|c|c|c|c|c|c|c|c|}
\hline$p_{T}^{a s s o c}$ range & $\left\langle v_{4}^{\text {trig }}\right\rangle$ & $\begin{array}{l}0-10 \% \\
\left\langle v_{4}^{a s s o c}\right\rangle\end{array}$ & $\left\langle v_{4}^{\text {trig }} v_{4}^{\text {assoc }}\right\rangle$ & $\left\langle v_{4}^{\text {trig }}\right\rangle$ & $\begin{array}{l}20-40 \% \\
\left\langle v_{4}^{a s s o c}\right\rangle\end{array}$ & $\left\langle v_{4}^{t r i g} v_{4}^{a s s o c}\right\rangle$ & $\left\langle v_{4}^{t r i g}\right\rangle$ & $\begin{array}{l}50-80 \% \\
\left\langle v_{4}^{\text {assoc }}\right\rangle\end{array}$ & $\left\langle v_{4}^{t r i g} v_{4}^{a s s o c}\right\rangle$ \\
\hline $0.2-0.4 \mathrm{GeV} / \mathrm{c}$ & 0.075574 & 0.000948 & 0.000221 & 0.030574 & 0.001625 & 0.000085 & -0.003253 & -0.001360 & 0.000677 \\
\hline 0.4-0.6 GeV/c & 0.075574 & 0.005678 & 0.000723 & 0.030574 & 0.004099 & 0.000683 & -0.003253 & -0.002469 & 0.000986 \\
\hline $0.6-0.8 \mathrm{GeV} / \mathrm{c}$ & 0.075574 & 0.012493 & 0.001632 & 0.030574 & 0.007566 & 0.001628 & -0.003253 & -0.002682 & 0.001783 \\
\hline $0.8-1.0 \mathrm{GeV} / \mathrm{c}$ & 0.075574 & 0.020305 & 0.002501 & 0.030574 & 0.011671 & 0.002133 & -0.003253 & -0.003266 & 0.002189 \\
\hline $1.0-1.2 \mathrm{GeV} / \mathrm{c}$ & 0.075574 & 0.028170 & 0.003525 & 0.030574 & 0.015414 & 0.003059 & -0.003253 & -0.002346 & 0.003384 \\
\hline $1.2-1.4 \mathrm{GeV} / \mathrm{c}$ & 0.075574 & 0.035320 & 0.004594 & 0.030574 & 0.018721 & 0.004194 & -0.003253 & -0.002007 & 0.002937 \\
\hline $1.4-1.6 \mathrm{GeV} / \mathrm{c}$ & 0.075574 & 0.042858 & 0.005170 & 0.030574 & 0.021235 & 0.004439 & -0.003253 & -0.002812 & 0.002656 \\
\hline $1.6-1.8 \mathrm{GeV} / \mathrm{c}$ & 0.075574 & 0.048819 & 0.005748 & 0.030574 & 0.025388 & 0.005695 & -0.003253 & -0.004228 & 0.001316 \\
\hline $1.8-2.0 \mathrm{GeV} / \mathrm{c}$ & 0.075574 & 0.053568 & 0.006991 & 0.030574 & 0.028373 & 0.006568 & -0.003253 & -0.005650 & 0.002296 \\
\hline $2.0-2.2 \mathrm{GeV} / \mathrm{c}$ & 0.075574 & 0.056891 & 0.006922 & 0.030574 & 0.029028 & 0.006538 & -0.003253 & -0.002869 & 0.005727 \\
\hline $2.2-2.4 \mathrm{GeV} / \mathrm{c}$ & 0.075574 & 0.058573 & 0.007499 & 0.030574 & 0.031020 & 0.005983 & -0.003253 & -0.000363 & 0.005555 \\
\hline
\end{tabular}

TABLE VII: $\left\langle v_{5}^{\text {trig }}\right\rangle\left\langle v_{5}^{a s s o c}\right\rangle\left\langle v_{5}^{\text {trig }} v_{5}^{\text {assoc }}\right\rangle$

\begin{tabular}{|c|c|c|c|c|c|c|c|c|c|}
\hline$p_{T}^{\text {assoc }}$ range & $\left\langle v_{5}^{\text {trig }}\right\rangle$ & $\begin{array}{l}0-10 \% \\
\left\langle v_{5}^{\text {assoc }}\right\rangle\end{array}$ & $\left\langle v_{5}^{\text {trig }} v_{5}^{\text {assoc }}\right\rangle$ & $\left\langle v_{5}^{\text {trig }}\right\rangle$ & $\begin{array}{l}20-40 \% \\
\left\langle v_{5}^{\text {assoc }}\right\rangle\end{array}$ & $\left\langle v_{5}^{\text {trig }} v_{5}^{a s s o c}\right\rangle$ & $\left\langle v_{5}^{\text {trig }}\right\rangle$ & $\begin{array}{l}50-80 \% \\
\left\langle v_{5}^{\text {assoc }}\right\rangle\end{array}$ & $\left\langle v_{5}^{\text {trig }} v_{5}^{a s s o c}\right\rangle$ \\
\hline $0.2-0.4 \mathrm{GeV} / \mathrm{c}$ & 0.035613 & 0.000163 & 0.000072 & -0.004876 & -0.000210 & 0.000189 & -0.006631 & -0.001752 & -0.000043 \\
\hline $0.4-0.6 \mathrm{GeV} / \mathrm{c}$ & 0.035613 & 0.001217 & 0.000029 & -0.004876 & -0.000709 & 0.000279 & -0.006631 & -0.003521 & -0.000147 \\
\hline $0.6-0.8 \mathrm{GeV} / \mathrm{c}$ & 0.035613 & 0.003841 & 0.000401 & -0.004876 & -0.000459 & 0.000549 & -0.006631 & -0.004073 & 0.000861 \\
\hline $0.8-1.0 \mathrm{GeV} / \mathrm{c}$ & 0.035613 & 0.006993 & 0.000565 & -0.004876 & -0.000890 & 0.000854 & -0.006631 & -0.004225 & 0.000594 \\
\hline $1.0-1.2 \mathrm{GeV} / \mathrm{c}$ & 0.035613 & 0.010640 & 0.001154 & -0.004876 & -0.000437 & 0.000669 & -0.006631 & -0.005513 & -0.000439 \\
\hline $1.2-1.4 \mathrm{GeV} / \mathrm{c}$ & 0.035613 & 0.014408 & 0.001556 & -0.004876 & -0.000367 & 0.000239 & -0.006631 & -0.005430 & 0.001545 \\
\hline $1.4-1.6 \mathrm{GeV} / \mathrm{c}$ & 0.035613 & 0.018030 & 0.001783 & -0.004876 & 0.000923 & 0.000809 & -0.006631 & -0.006749 & 0.001939 \\
\hline $1.6-1.8 \mathrm{GeV} / \mathrm{c}$ & 0.035613 & 0.020953 & 0.002676 & -0.004876 & 0.002621 & 0.001746 & -0.006631 & -0.007577 & 0.002326 \\
\hline $1.8-2.0 \mathrm{GeV} / \mathrm{c}$ & 0.035613 & 0.023771 & 0.002333 & -0.004876 & 0.001566 & 0.001071 & -0.006631 & -0.005370 & 0.001255 \\
\hline $2.0-2.2 \mathrm{GeV} / \mathrm{c}$ & 0.035613 & 0.027007 & 0.003120 & -0.004876 & 0.001490 & 0.002077 & -0.006631 & -0.011794 & 0.001494 \\
\hline $2.2-2.4 \mathrm{GeV} / \mathrm{c}$ & 0.035613 & 0.030243 & 0.002670 & -0.004876 & 0.003509 & 0.002326 & -0.006631 & -0.011982 & 0.001287 \\
\hline
\end{tabular}

TABLE VIII: $\left\langle v_{6}^{\text {trig }}\right\rangle\left\langle v_{6}^{a s s o c}\right\rangle\left\langle v_{6}^{\text {trig }} v_{6}^{\text {assoc }}\right\rangle$

\begin{tabular}{|c|c|c|c|c|c|c|c|c|c|}
\hline$p_{T}^{a s s o c}$ range & $\left\langle v_{6}^{t r i g}\right\rangle$ & $\begin{array}{l}0-10 \% \\
\left\langle v_{6}^{\text {assoc }}\right\rangle\end{array}$ & $\left\langle v_{6}^{t r i g} v_{6}^{a s s o c}\right\rangle$ & $\left\langle v_{6}^{t r i g}\right\rangle$ & $\begin{array}{l}20-40 \% \\
\left\langle v_{6}^{\text {assoc }}\right\rangle\end{array}$ & $\left\langle v_{6}^{\text {trig }} v_{6}^{a s s o c}\right\rangle$ & $\left\langle v_{6}^{t r i g}\right\rangle$ & $\begin{array}{l}50-80 \% \\
\left\langle v_{6}^{\text {assoc }}\right\rangle\end{array}$ & $\left\langle v_{6}^{t r i g} v_{6}^{a s s o c}\right\rangle$ \\
\hline $0.2-0.4 \mathrm{GeV} / \mathrm{c}$ & 0.014519 & -0.000231 & 0.000120 & -0.013741 & 0.000010 & -0.000044 & -0.001509 & 0.000120 & -0.000396 \\
\hline $0.4-0.6 \mathrm{GeV} / \mathrm{c}$ & 0.014519 & 0.000030 & 0.000215 & -0.013741 & -0.000491 & -0.000084 & -0.001509 & -0.001390 & -0.000110 \\
\hline $0.6-0.8 \mathrm{GeV} / \mathrm{c}$ & 0.014519 & 0.000156 & -0.000083 & -0.013741 & -0.001263 & -0.000056 & -0.001509 & -0.001474 & -0.000271 \\
\hline $0.8-1.0 \mathrm{GeV} / \mathrm{c}$ & 0.014519 & 0.001058 & 0.000036 & -0.013741 & -0.002195 & 0.000216 & -0.001509 & 0.000550 & 0.000498 \\
\hline $1.0-1.2 \mathrm{GeV} / \mathrm{c}$ & 0.014519 & 0.002324 & 0.000420 & -0.013741 & -0.002858 & 0.000355 & -0.001509 & -0.000728 & 0.000465 \\
\hline $1.2-1.4 \mathrm{GeV} / \mathrm{c}$ & 0.014519 & 0.003721 & 0.000349 & -0.013741 & -0.003420 & 0.000420 & -0.001509 & -0.002572 & -0.000688 \\
\hline $1.4-1.6 \mathrm{GeV} / \mathrm{c}$ & 0.014519 & 0.004106 & 0.000447 & -0.013741 & -0.003858 & 0.000966 & -0.001509 & -0.002692 & 0.000093 \\
\hline $1.6-1.8 \mathrm{GeV} / \mathrm{c}$ & 0.014519 & 0.006095 & 0.000886 & -0.013741 & -0.005499 & 0.000622 & -0.001509 & -0.002420 & 0.002307 \\
\hline $1.8-2.0 \mathrm{GeV} / \mathrm{c}$ & 0.014519 & 0.007962 & 0.001136 & -0.013741 & -0.004095 & 0.000763 & -0.001509 & -0.002748 & 0.001520 \\
\hline $2.0-2.2 \mathrm{GeV} / \mathrm{c}$ & 0.014519 & 0.007611 & -0.000213 & -0.013741 & -0.001545 & 0.000804 & -0.001509 & 0.002193 & -0.000750 \\
\hline $2.2-2.4 \mathrm{GeV} / \mathrm{c}$ & 0.014519 & 0.008142 & 0.000370 & -0.013741 & -0.001668 & 0.000705 & -0.001509 & 0.003055 & 0.000971 \\
\hline
\end{tabular}

